\title{
Three-dimensional echocardiography of tricuspid valve for visualizing all the three leaflets
}

Raj Kumar, MD and Rahul Mehrotra, MD

Kumar R, Mehrotra R. Three-dimensional echocardiography of tricuspid valve for visualizing all the three leaflets. Curr Res Cardiol 2017;4(3):35

Three-dimensional echocardiography offers a unique opportunity of visualizing all the three leaflets of tricuspid valve in en-face view from transthoracic windows. It is very useful in localizing the position of the pacing leads and for planimetry (Figures 1 and 2).
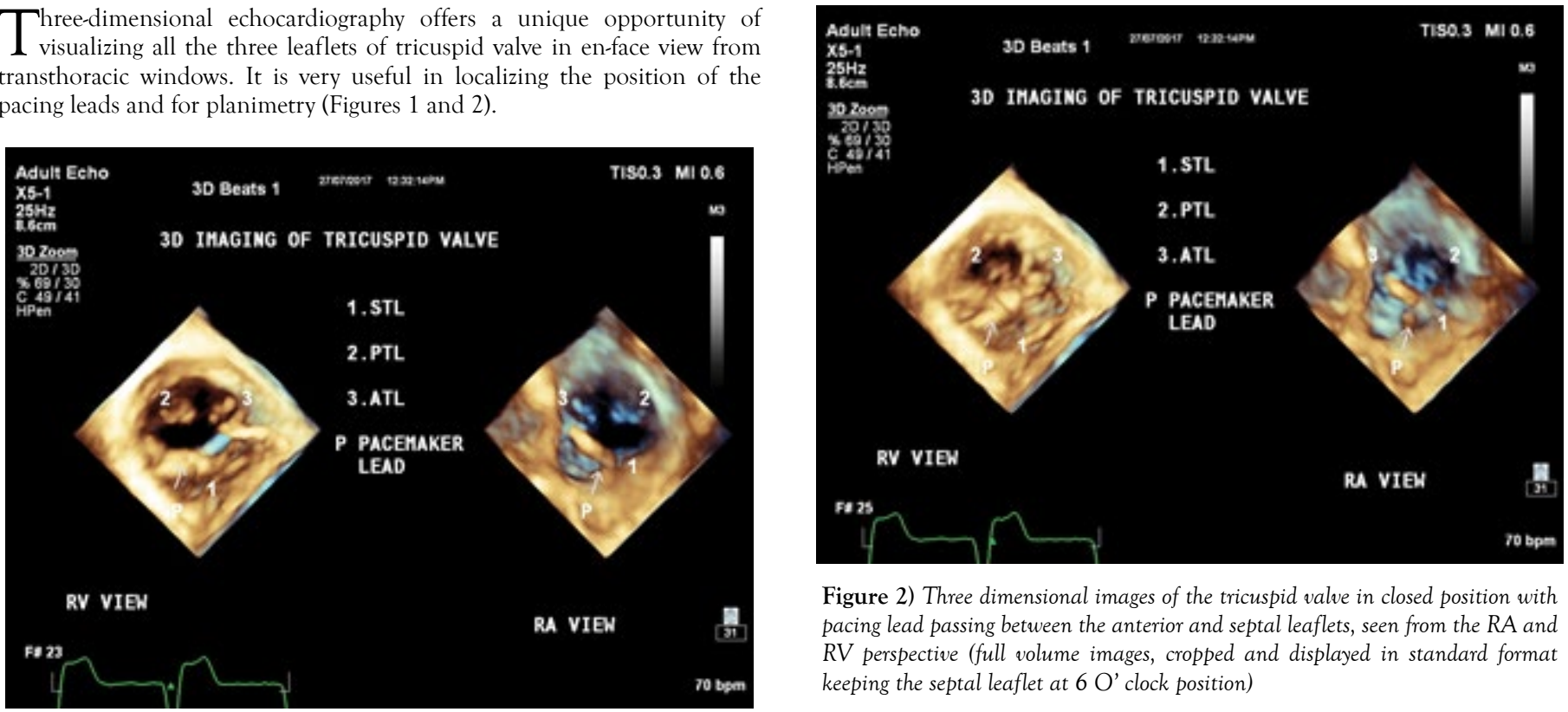

Figure 2) Three dimensional images of the tricuspid valve in closed position with pacing lead passing between the anterior and septal leaflets, seen from the RA and $R V$ perspective (full volume images, cropped and displayed in standard format keeping the septal leaflet at 6 O' clock position)

Figure 1) Three dimensional images of the tricuspid valve in open position with pacing lead passing between the anterior and septal leaflets, seen from the $R A$ and $R V$ perspective (full volume images, cropped and displayed in standard format keeping the septal leaflet at 6 O' clock position)

Non-Invasive Cardiology, Max Super Speciality hospital, Saket, New Delhi, India

Correspondence: Dr Rahul Mehrotra, MD, Non-Invasive Cardiology, Max Super Speciality hospital, Saket, New Delhi, India, Telephone 01126515050 , e-mail Rahul. Mehrotra@maxhealthcare.com

Received: August 21, 2017, Accepted: August 22, 2017, Published: August 24, 2017 